\title{
Machine learning in electricity fraud detection in smart grids with multivariate Gaussian distribution
}

\author{
Simona-Vasilica OPREA \\ Bucharest University of Economic Studies, Bucharest, Romania \\ simona.oprea@csie.ase.ro \\ Adela BÂRA \\ Bucharest University of Economic Studies, Bucharest, Romania \\ bara.adela@ie.ase.ro \\ Niculae OPREA \\ SC ICPE S.A., Bucharest, Romania \\ nicu_oprea1982@yahoo.com
}

\begin{abstract}
Smart meters allow electricity consumption readings at a high time resolution generating time series that can be investigated to extract valuable insights and detect frauds. Using a dataset with recordings from Chinese consumers, we propose an exploratory data analysis and processing to train several classifiers and assess the results. Good results are obtained with ensemble classifiers such as Random Forest (RF), eXtreme Gradient Boosting (XGB) and Multi-Layer Perceptron (MLP) with two layers and a relatively small number of neurons. Real-consumption dataset daily recorded in China consisting of over 42,000 consumers and over 1,000 days is processed with machine learning ML algorithms or classifiers to distinguish between normal and suspicious consumers. In this paper, we will compare a simple feature engineering method that consists in aggregating the data, calculating distances and density function with no feature engineering, proving that the first approach enhances the results and reduces the utility companies' costs related to on-site inspections. The results are compared with AUC score and ROC curves as the input data is highly skewed.
\end{abstract}

Keywords: First keyword, second keyword, third keyword, forth keyword, fifth keyword.

\section{Introduction}

With conventional meters, the suspicious consumers normally consume half of the month while the other half the meter is bypassed so that on average the consumption is constant over time. However, this way of stealing is no longer met very often with smart meters, as they allow frequent readings that reveal the consumption irregularities over time. Also, the challenge in detecting electricity frauds with smart-metered data consists of continuously stealing of some of the consumed electricity that is difficult to identify.

Fraud detection is a continuous challenge regardless the metering type (with conventional or smart meters) because of the difficulty in detecting the suspicious consumers. Training of the model is actually difficult as the data may have numerous issues from the classification point of view such as missing and unbalanced data. Therefore, for missing data several methods are implemented such as interpolation, back- and forward-fill as in Figure 1 whereas for balancing the training dataset, SMOTE or ADASYN are preferred. It can be noticed that in 2005 many data are missing. The reason for missing data can be a reading problem of the meter or a tempering. It can be notice that the consumption pattern or the bandwidth in 2014 differs from the consumption pattern in 2016 that is characterized by a much higher variability. 


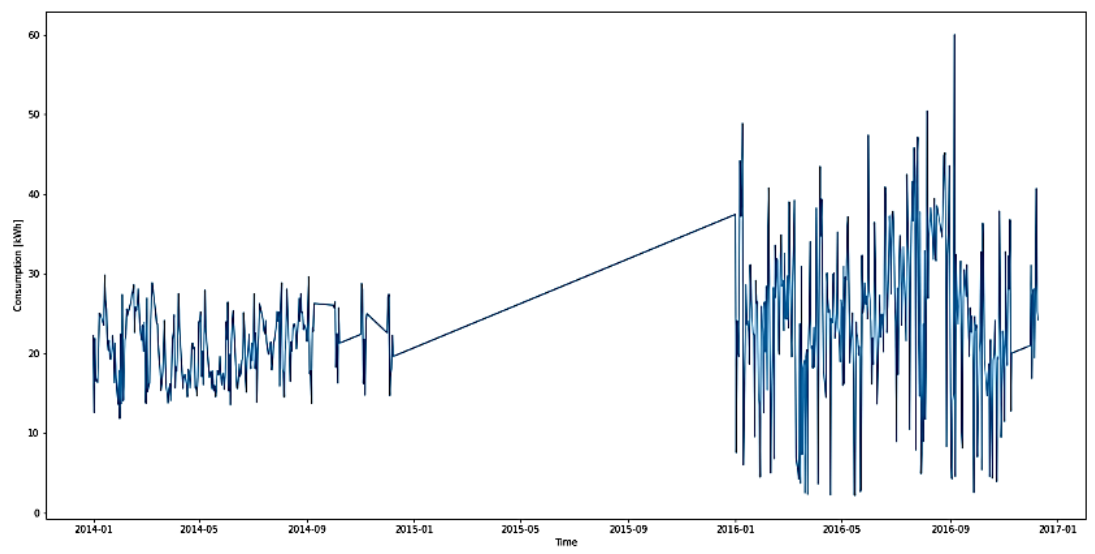

PICBE |

544

The recordings are provided by the Chinese utility company and they span from January 2014 until September 2016 containing 42,372 consumers and 1,034 daily time series. Considering the high number variables, only few time-series for several consumers are plotted in Figure 2.

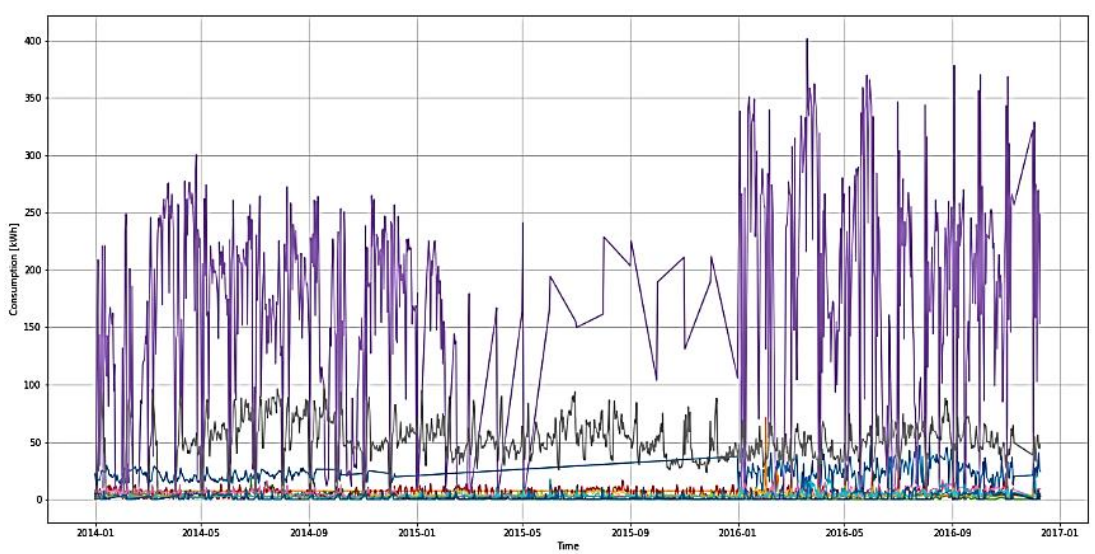

Figure 2. Daily consumption time series plot

In Figure 2, it can be noticed that in 2015 numerous data is missing. Moreover, the data is characterised by seasonality and noise. The percentage of missing data on 3/10/2014 is over $99.96 \%$, whereas other days may record a percentage of below $50 \%$.

\section{Scientific literature}

Various studies were proposed for fraud detection in electricity consumption recorded by conventional or smart meters. Most of the research studies were performed with open-source data provided by utility companies or regulation authorities from China, Tunisia, Uruguay, Serbia, Ireland, Spain, Brazil, etc. (Coma-Puig, Carmona, Gavalda, Alcoverro, \& Martin, 2016), (Spirić, Dočić, \& Stanković, 2015), (Zanetti et al., 2019), (Yip, Tan, Tan, Gan, \& Wong, 2018).

The research methods are split between supervised ML algorithms, classical (Cody, Ford, \& Siraj, 2016) and unsupervised ML algorithms (Jain, Choksi, \& Pindoriya, 2019). The chosen approach depends on the target availability and characteristics of data such as skewness. 
The financial losses caused by the electricity frauds are significant and could be lowered by several measures such as ML data analytics and on-site inspections. However, both measures lead to costs and benefits that can be assessed (Massaferro, Martino, \& Fernandez, 2020).

A study based on distances is presented in (Basu, Debusschere, Douzal-Chouakria, \& Bacha, 2015) analysing time series datasets recorded in residential building with a non-intrusive approach.

Another research proposed empirical mode decomposition and K-Nearest Neighbors classifier using the same dataset provided by the Chinese utility company State Grid Corporation of China. Time series feature extraction is implemented and the unbalanced dataset is oversampled with ADASYN (Aziz, Naqvi, Khan, \& Aslam, 2020).

Emerging IoT and fog are applied for analysing data steams to identify the irregularities in consumption and carry out verifications eliminating the time gaps between the detection and inspection (Siffer, Fouque, Termier, \& Largouet, 2017), (Lyu, Jin, Rajasegarar, He, \& Palaniswami, 2017).

\section{Methodology}

Two approaches or scenarios are considered in this paper: no feature engineering (scenario1) and simple feature engineering (scenario2) grouping and aggregating the data, calculating new features with distance and density function, and. Hence, the dataset is analysed, and up to a point similar pre-processing steps are applied for the two approaches. First, the dataset is transposed because the consumers are listed as rows and daily consumption as columns. Therefore, for data pre-processing, the dataset is transposed, flag is removed and then the following pre-processing steps are applied:

- For daily consumption sorting and data formatting are required;

- For missing data, we used interpolation and back- and forward-fill methods;

- Noise reduction with Savgol filter from SciPy library. Also, Savitzky-Golay filter is implemented but the results were not better.

Second, the pre-processed dataset is transposed again so that the features to be daily consumption and the flag is reinserted.

For an improved Gaussian distribution of the features, $\log$ function is applied to $X$. Also, MinMax scaling of $X$ dataset is applied as the most classifiers performs better and faster when the input data is scaled. Usually, a standard or a robust scaler that removes outliers could be alternatively applied. The methodology is briefly presented in Figure 3.

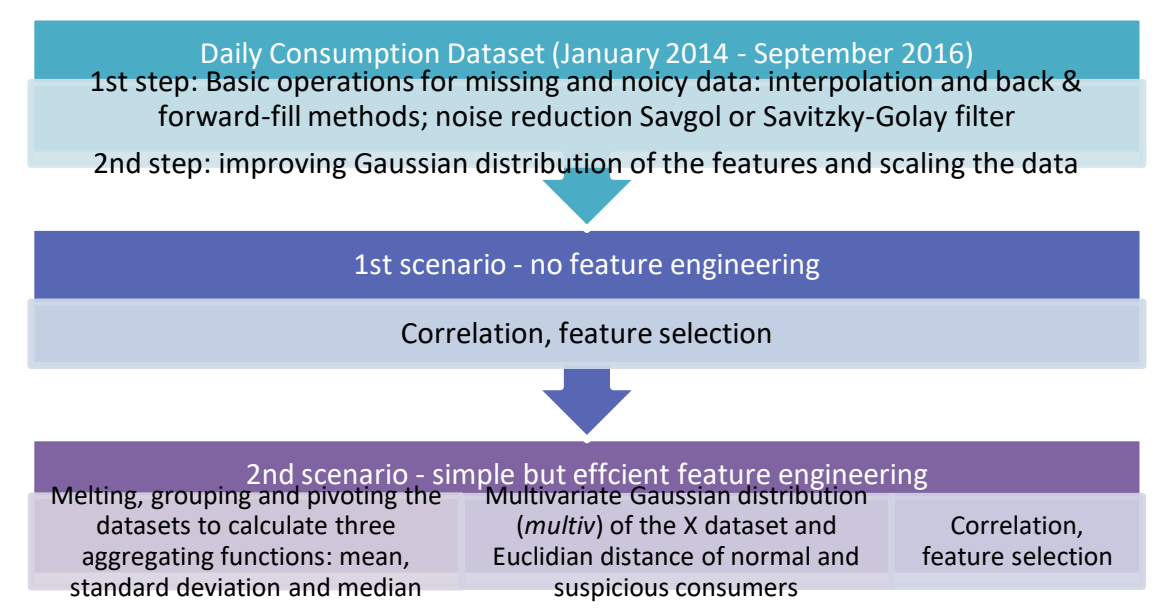

Figure 3. Consumption data processing methodology

DOI: 10.2478/picbe-2021-0049, pp. 543-551, ISSN 2558-9652|

Proceedings of the $15^{\text {th }}$ International Conference on Business Excellence 2021 
The dataframe $d f$ dimensionality will be reduced grouping and aggregating the features load by mean, median and standard deviation aggregate functions $a g g_{f}$. Hence, a reduced datasets $a g g \_d f$ will be obtained. The scope of calculating aggregate features is to compare them with the details and identify anomalies.

$$
\begin{aligned}
& d f . g_{r o u p b y}\left(l_{\text {load }}\right) \cdot \mathrm{agg}_{f} \rightarrow a g g_{-} d f \\
& a g g_{f}=\{\text { mean, median, std }\}
\end{aligned}
$$

Also, the dataset can be grouped by days of the week (from 0 - Monday to 6 - Sunday) and we will obtained extra features.

$$
d f . g r o u p b y\left(\text { weekday). } a g g_{f} \rightarrow a g g_{-} d f_{-} w d\right.
$$

A new dataframe $d f$ will be created by merging the two sets of features.

$d f=j o i n\left(a g g_{-} d f, a g g_{-} d f_{\_} w d\right)$

The target $y$ will be extracted from $d f$ then defining $X$ is the next step.

$$
\begin{aligned}
& y=d f(\text { flag }) \\
& X=d f-d f(\text { flag })
\end{aligned}
$$

$X$ is scaled as this process enhances the performance of classifiers and improves the convergence speed.

$$
\begin{aligned}
& \text { scaled_X }=\text { scaler }(X) \\
& \text { scaler } \in\{\text { MinMax,Standard, Robust, Normalizer }\}
\end{aligned}
$$

The best features are selected for performant training.

$$
\text { feature_selection }(\text { scaled_X,y) } \rightarrow \text { reduced_X }
$$

The reduced $X$ set and $\mathrm{y}$ will be split into training and testing sets.

$$
\text { split(reduced_X,y) } \rightarrow X_{\text {train }}, y_{\text {train }}, X_{\text {test }}, y_{\text {test }}
$$

The datasets $X_{\text {train }}, y_{\text {train }}$ are balanced using SMOTE or ADASYN and a sampling strategy ratio.

$$
\text { sampling }\left(X_{\text {train }}, y_{\text {train }}\right) \rightarrow X_{\text {train }}^{\text {balanced }}, y_{\text {train }}^{\text {balanced }}
$$

The model selection and metrics are described in the following paragraphs. For training the balanced datasets $X_{\text {train }}^{\text {balanced }}, y_{\text {train }}^{\text {balanced }}$, we implement ten ML classifiers to obtain $y_{\text {predict }}$ :

$\operatorname{train}\left(M L\left(X_{\text {train }}^{\text {balanced }}, y_{\text {train }}^{\text {balanced }}\right)\right) \rightarrow M L\left(X_{\text {test }}\right)$

$M L\left(X_{\text {test }}\right) \rightarrow y_{\text {predict }}$

The following metrics are useful for proving the performance of the classifiers:

metrics $=\{$ accuracy, precision, recall, F1 score, AUC and ROC - AUC score $\}$ (15)

Accuracy is significant when the flag or the target $y$ is well-balanced. Otherwise, the other metrics should be calculated. Plotting learning curves and validation curves is useful to improve the performance of the classifiers. 


\section{Scenario1 - no feature engineering}

44 features based on daily consumption are weakly and positively correlated with the flag and the Pearson correlation coefficients range between 0.12 and 0.1 . The rest of the features are even lower correlated with the target. The correlation map is presented in Figure 4.

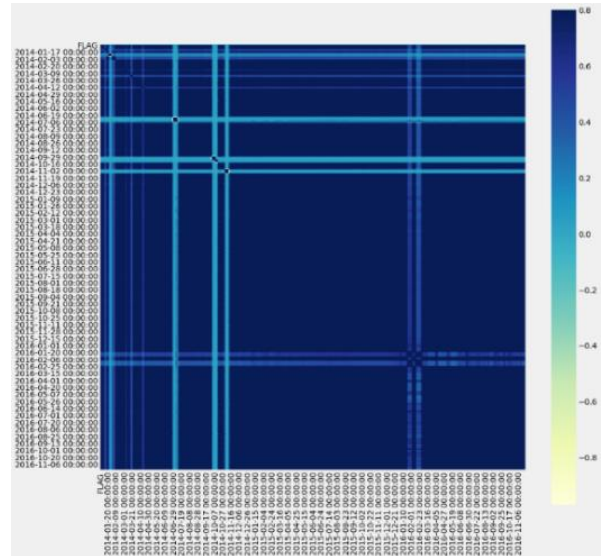

PICBE |

Figure 4. Heatmap for 1st scenario

\section{Scenario2 - simple feature engineering}

Regarding the simple feature engineering method, we proposed to perform melting, grouping and pivoting the datasets to calculate three aggregating functions: mean, standard deviation and median. Interesting insights regarding difference between normal consumers and suspicious consumers are extracted from the processed dataset. By melting the dataset, the consumers and dates are placed as rows and the daily consumption becomes a column on which aggregation functions can be applied. Grouping the data by weekday allows us to compute mean, standard deviation and median for each weekday. Therefore, 7 by 3 features are created. Also, the aggregation functions are applied without grouping the data by weekday resulting another three features.

Additional features are calculated as multivariate Gaussian distribution (multiv) of the $\mathrm{X}$ dataset and Euclidian distance of normal and suspicious consumers. Such features have the purpose to better identifying the suspicious consumers. Still, the features are weakly positively/negatively correlated with the flag and the Pearson correlation coefficients range between -0.19 and 0.08 . The correlation map is presented in Figure 5.

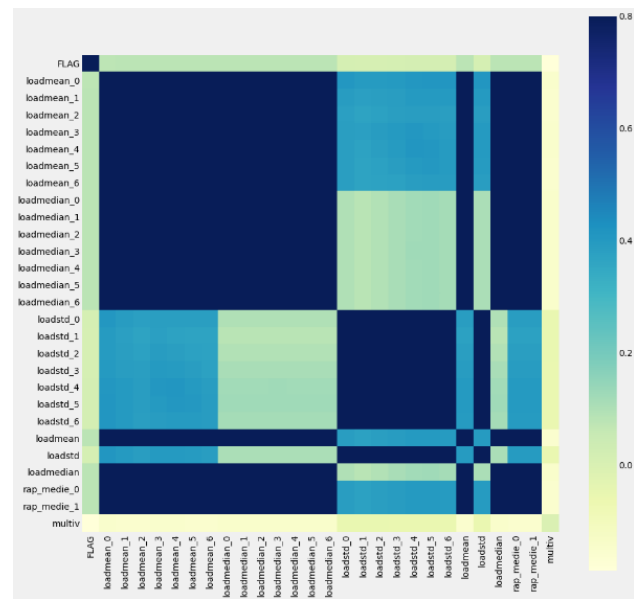

Figure 5. Heatmap for 2nd scenario 


\section{Simulation and results}

The consumption dataset reveals the following characteristics for the two categories of consumers (FLAG $=0 /$ normal and FLAG $=1$ /suspicious). The results are obtained for September 2014, but the trends are similar in the rest of the months. The mean consumption on weekday and week number is presented in Figure 6.
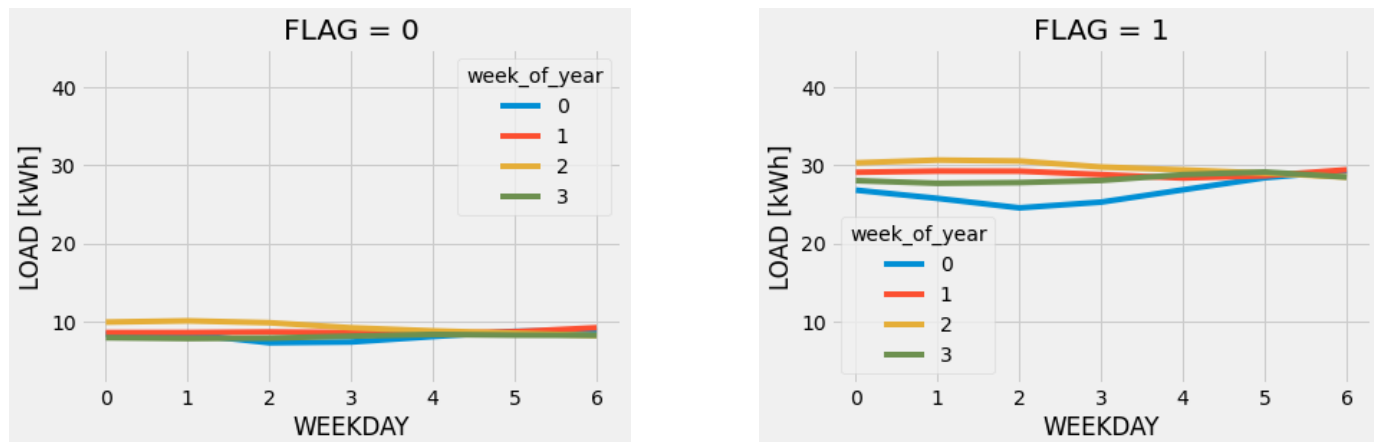

Figure 6. Mean consumption per weekday and week number of the month

Furthermore, the two categories of consumers differ from the consumption variance point of view as in Figure 7.
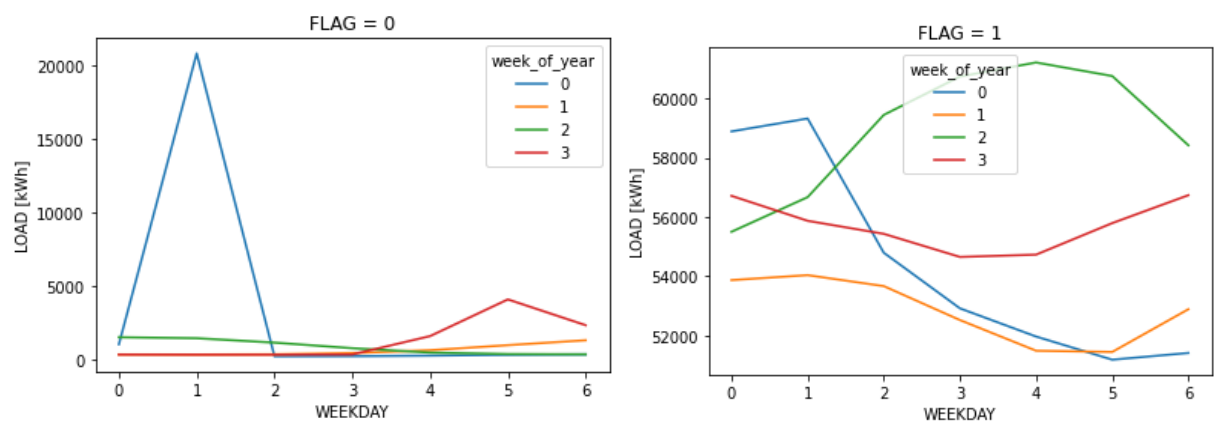

Figure 7. Variance of the consumption data

Analysis the autocorrelation, both categories show strong autocorrelation with the first 2 previous days, also PACF indicates autoregressive component especially in case of normal consumers considering the spikes that are out of the light blue area.
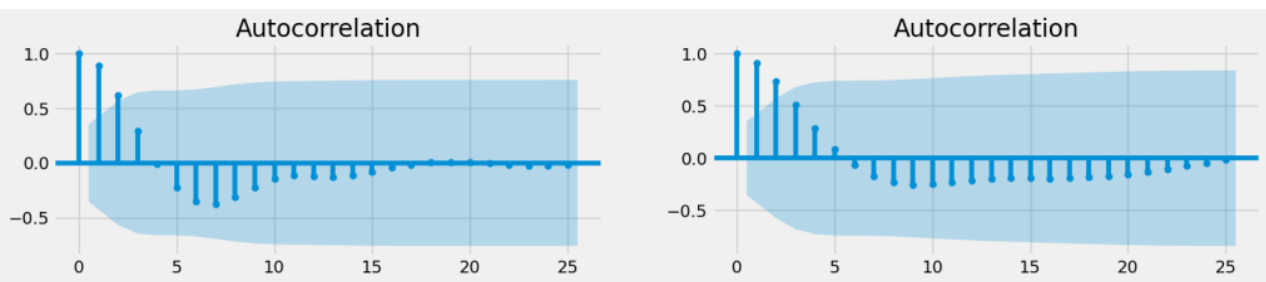

Figure 8. ACF for (a) normal consumers - left and (b) suspicious consumers - right 

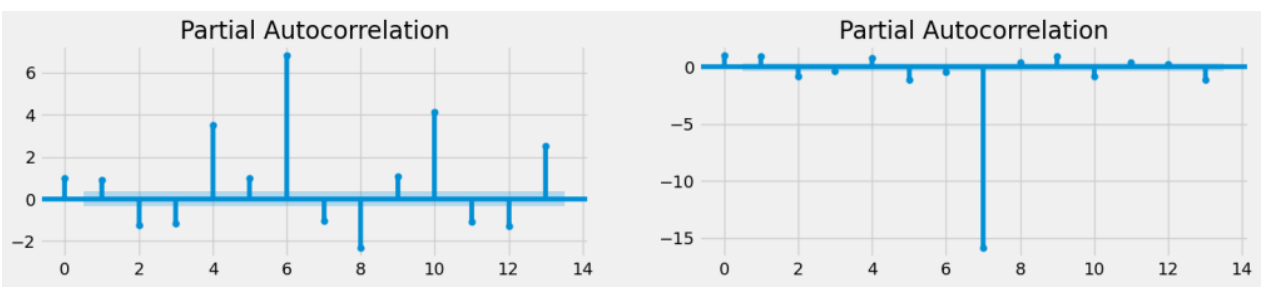

Figure 9. PACF for (a) normal consumers - left and (b) suspicious consumers - right

Some other interesting characteristics of normal and suspicious consumers are revealed in Figure 10.
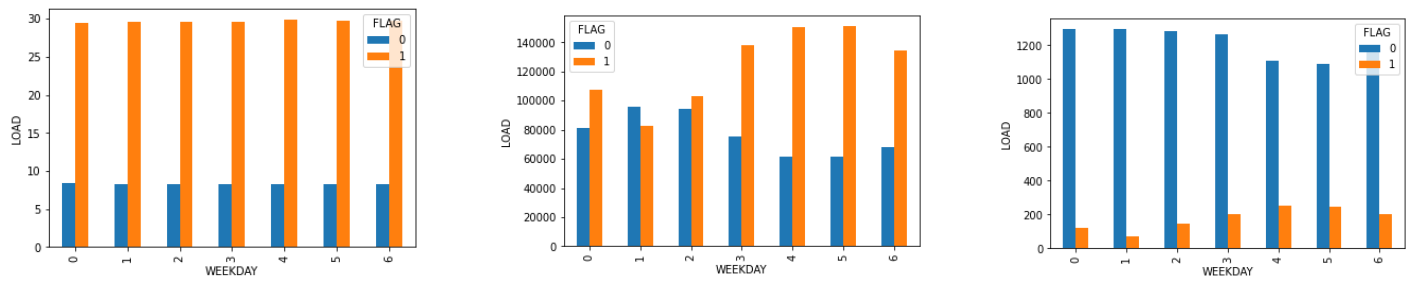

Figure 10. Consumption mean (left), variance (middle) and skewness (right)

Consumption mean and variance of the suspicious consumers are usually bigger than those recorded for normal consumers. One exception is found on Tuesdays. Whereas the consumption data is more skewed for normal consumers.

\section{1st Scenario}

The best 34 features are selected for training the model. Apart from these three algorithms, another 7 are applied to detect suspicions consumers (Linear Regression - LR, Stochastic Gradient Descend - SGD, Decision Tree - DT, Light Gradient Boosting - LGB, Quadratic Discriminant Analysis QDA, K-nearest Neighbour - KN, Ada Boost - AB).

Splitting the dataset into train and test datasets is performed identical for the two approaches. Confusion matrixes for the best three algorithms (MLP, RF, XGB) are presented in Figure 11. The best ROC-AUC score without feature engineering is 0.7 which show a rather modest performance.
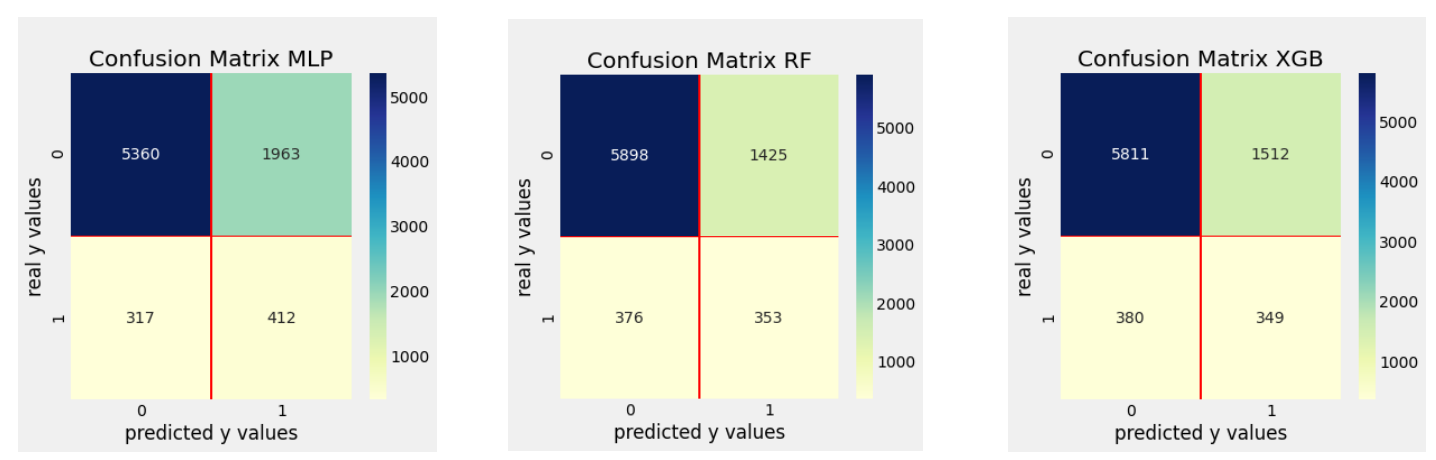

Figure 11. Confusion matrices - 1st scenario

\section{2nd Scenario}

In the second scenario, the best ROC-AUC score is 0.76 . The confusion matrices are presented in Figure 12. 

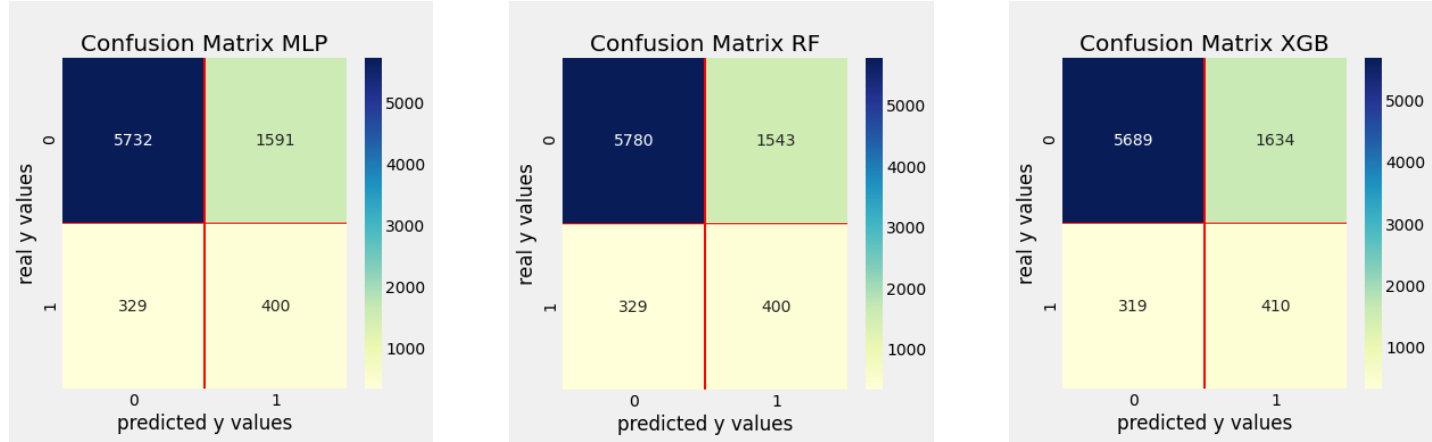

PICBE |

550

The ROC curves for the two approaches are showed in Figure 13.
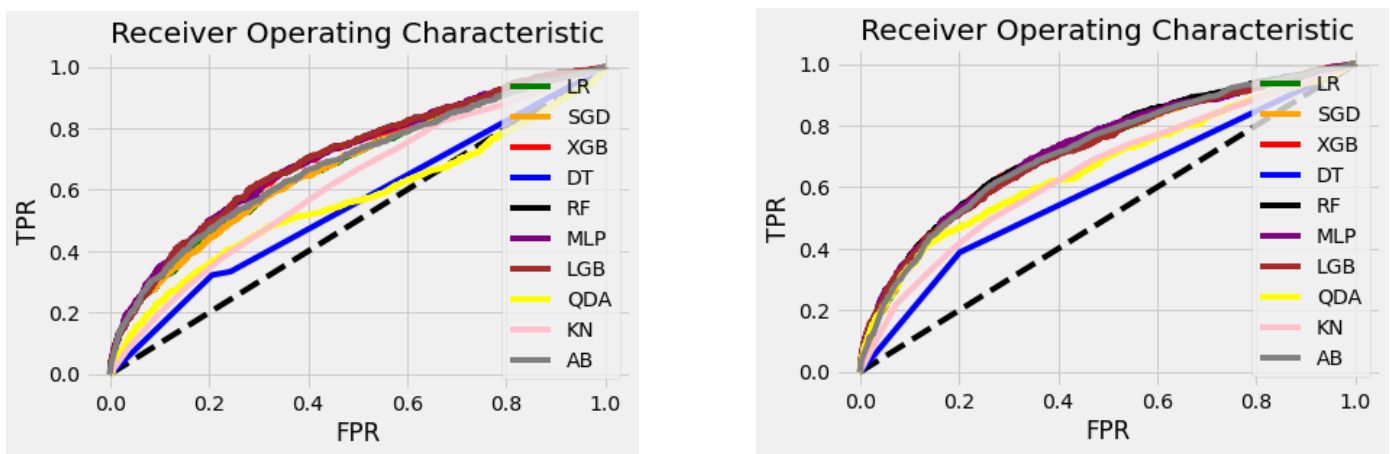

Figure 13. ROC curves for the two approaches

Even though the results are not astonishing improved, the higher ROC-AUC the lower the costs encounter the utility companies for supplementary inspections.

\section{Conclusions}

In this paper, we proposed a simple but efficient feature engineering approach that enhance the results of the supervised algorithms adding new features related to the multivariate Gaussian distribution and Euclidian distances for the two types of consumers normal and suspicious. The ROC-AUC curves improved the metric from 0.7 to 0.76 that reduces the costs of the utility companies in identifying frauds in electricity consumption.

In this sense, we proposed a methodology to process the input data stating from basic preprocessing, processing and training of the model. The future research will focus on enhancing feature engineering for time series that are offered as useful packages, such as tsfel offered as a Python library.

\section{Acknowledgment}

This work was supported by a grant of the Romanian Ministry of Research and Innovation, CCCDI UEFISCDI, project number 462PED/28.10.2020, project code PN-III-P2-2.1-PED-2019-1198, within PNCDI III. 


\section{References}

Aziz, S., Naqvi, S. Z. H., Khan, M. U., \& Aslam, T. (2020). Electricity Theft Detection using Empirical Mode Decomposition and K-Nearest Neighbors. In 2020 International Conference on Emerging Trends in Smart Technologies, ICETST 2020. https://doi.org/10.1109/ICETST49965.2020.9080727.

Basu, K., Debusschere, V., Douzal-Chouakria, A., \& Bacha, S. (2015). Time series distance-based methods for non-intrusive load monitoring in residential buildings. Energy and Buildings. https://doi.org/10.1016/j.enbuild.2015.03.021.

Cody, C., Ford, V., \& Siraj, A. (2016). Decision tree learning for fraud detection in consumer energy consumption. In Proceedings - 2015 IEEE 14th International Conference on Machine Learning and Applications, ICMLA 2015. https://doi.org/10.1109/ICMLA. 2015.80.

Coma-Puig, B., Carmona, J., Gavalda, R., Alcoverro, S., \& Martin, V. (2016). Fraud detection in energy consumption: A supervised approach. In Proceedings - 3rd IEEE International Conference on Data Science and Advanced Analytics, DSAA 2016. https://doi.org/ 10.1109/DSAA.2016.19.

Jain, S., Choksi, K. A., \& Pindoriya, N. M. (2019). Rule-based classification of energy theft and anomalies in consumers load demand profile. IET Smart Grid. https://doi.org/10.1049/ietstg.2019.0081.

Lyu, L., Jin, J., Rajasegarar, S., He, X., \& Palaniswami, M. (2017). Fog-empowered anomaly detection in IoT using hyperellipsoidal clustering. IEEE Internet of Things Journal. https://doi.org/10.1109/JIOT.2017.2709942.

Massaferro, P., Martino, J. M. Di, \& Fernandez, A. (2020). Fraud Detection in Electric Power Distribution: An Approach That Maximizes the Economic Return. IEEE Transactions on Power Systems. https://doi.org/10.1109/TPWRS.2019.2928276.

Siffer, A., Fouque, P. A., Termier, A., \& Largouet, C. (2017). Anomaly detection in streams with extreme value theory. In Proceedings of the ACM SIGKDD International Conference on Knowledge Discovery and Data Mining. https://doi.org/10.1145/3097983.3098144.

Spirić, J. V., Dočić, M. B., \& Stanković, S. S. (2015). Fraud detection in registered electricity time series. International Journal of Electrical Power and Energy Systems. https://doi.org/ 10.1016/j.ijepes.2015.02.037.

Yip, S. C., Tan, W. N., Tan, C. K., Gan, M. T., \& Wong, K. S. (2018). An anomaly detection framework for identifying energy theft and defective meters in smart grids. International Journal of Electrical Power and Energy Systems. https://doi.org/10.1016/j.ijepes. 2018.03.025.

Zanetti, M., Jamhour, E., Pellenz, M., Penna, M., Zambenedetti, V., \& Chueiri, I. (2019). A Tunable Fraud Detection System for Advanced Metering Infrastructure Using Short-Lived Patterns. IEEE Transactions on Smart Grid. https://doi.org/10.1109/TSG.2017.2753738. 